\title{
3D laser scanning and digital restoration of an archaeological find
}

\author{
Stergios Fragkos ${ }^{1}$, Emanuel Tzimtzimis ${ }^{1}$, Dimitrios Tzetzis $^{1 *}$, Oana Dodun ${ }^{2}$ and Panagiotis \\ Kyratsis $^{3}$ \\ ${ }^{1}$ International Hellenic University, 14km Thessaloniki - N. Moudania, School of Science and \\ Technology, Thermi GR57001, Greece \\ ${ }^{2}$ Gheorghe Asachi Technical University of Iasi, Department of Manufacturing Engineering, Iași \\ 700050, Romania \\ ${ }^{3}$ Western Macedonia University of Applied Sciences, Department of Mechanical Engineering and \\ Industrial Design, Kila Kozani, GR50100, Greece
}

\begin{abstract}
The current paper demonstrates the digital recreation and 3D printing of a missing fragment of an ancient ceramic pottery following digitization using a three dimensional laser scanning. The resulting pointcloud of the laser scans was treated with a series of advanced software for the creation of surfaces and ultimately for a digital model. An analytical methodology is presented revealing the step by step approach, which is an innovative way of recreating a missing fragment. Such approach aims to demonstrate the level of contribution that the ever evolving computer based technologies and 3D printing could bring to cultural heritage. The reverse engineering method presented for the reconstruction of a ceramic pottery, which is a part of the larger field of digital archaeology, is believed to benefit a variety of interested parties including 3D CAD users and designers, archaeologists and museum curators.
\end{abstract}

\section{Introduction}

Over the last years, reverse engineering had a rapid expand in different fields and became one of the most widely used methods for the reconstruction of archaeological finds. 3D scanning data enables damaged and missing parts to be previewed virtually in advance. Complex geometry and free-style surfaces require professionals to produce original scaled models for 3D scanning and manipulate the data for digital models [1, 2]. 3D printing as a rapid and accurate method, reduces the dependence upon lengthy, repetitive and tedious manual restoration work. For the reconstruction of a missing fragment there are three initial different phases. The first one called collection of data or data capturing, while the next phases are known as data processing and downstream applications [3]. A fourth step is the digital fabrication of the part by $3 \mathrm{D}$ printing. There are numerous examples of the usage of reverse engineering for different archaeological study cases [4-7].

\footnotetext{
* Corresponding author: d.tzetzis@ihu.edu.gr
} 
The current work aims to demonstrate an innovative way of recreating a missing fragment of an archaeological find using entirely 3D technologies. A pottery made of clay, was chosen for the scanning and reconstruction process, since it has a lot of missing parts as discovered from excavation with only limited number of bonded fragments attached together to reveal the basic design of the pottery. It was very important, to have the minimum contact with the bonded fragments of the pottery for restoration. The work focuses on a special pottery type called terra sigillata, which as an archaeological term that describes a specific type of simple and decorated tableware made in Italy and in Gaul (France and the Rhineland) during the Roman Times. Such ceramic pottery has shining surface slips ranging from a soft polish to a marvellous glaze-like shine, in a characteristic colour range from light orange to bright red; they were manufactured in standard shapes and sizes. They were also manufactured on an industrial scale and were widely exported. A desktop laser scanner was utilised in order to scan the pottery and to generate digital data so to describe the highly complex geometry of the scanned object and recreate a missing part. Advanced processing of these digital data was achieved as well as conversion to the corresponding format for modelling and 3D printing, materializing in this way the digital created model.

\section{Laser scanning \& CAD process}

For the 3D scanning of archaeological finds, a system with a relatively high accuracy is required. The whole idea here is to choose an instrument that is capable of detecting the amount of geometric variation present in a given characteristic. The highly accurate NextEngine 3D laser scanner was used for the scanning procedure. The laser scanner contains two arrays of four solid state lasers, two 3.0 megapixel cameras and two lights for image capture. The laser scanner could scan objects of field size between $129.5 \mathrm{~mm}$ x 96.5 $\mathrm{mm}$ and $343 \mathrm{~mm} \times 256.5 \mathrm{~mm}$. It could provide texture density of $150 \mathrm{dpi}$ up to $400 \mathrm{dpi}$ and its dimensional accuracy lied between, + or $-0.127 \mathrm{~mm}$ and + or $-0.38 \mathrm{~mm}$ with acquisition speed of 50,000 points per second. The ScanStudio software was utilized for the data collection and initial processing. Additionally, the Geomagic Studio software, as well as the Solidworks parametric CAD software were used for the further processing of the CAD model.

\section{Reverse engineering workflow}

\subsection{Scanning}

The setup of the scanner was arranged in that way that the scanned object was placed approximately $430 \mathrm{~mm}$ from the scanner, achieving accuracy of $0.38 \mathrm{~mm}$ and field of view $254 \mathrm{~mm} \times 330 \mathrm{~mm}$. The scanned object was placed on different positions as shown in Figure 1. It was positioned two times vertically in order to overcome the phenomenon of self-occlusion.

The ceramic pottery was placed on a rotating base in front of the scanner. Vertical laser arrays reflect on the sample to be scanned, giving information about its geometry and texture. The data acquisition method for this scanner is laser light triangulation. The scanner can scan the object while it is steady. The sample rotates for a predefined number of degrees. In the current case this was set $36^{\circ}$ being seen through preliminary tests that were provided sufficient points to reconstruct the surface. The number of scans that occurs were aligned sequentially. 


\subsection{Point cloud processing}

The outcome of the three scans was a point cloud that needed further processing so to eliminate the extra irrelevant with the pottery, geometries such as the support stand, as illustrated in Figure 2. After the trimming, the alignment of the three scans was made by using the appropriate reference points (coloured pins). Alignment is the process where one object is moved into alignment with another one so that they both share the same coordinate system. The alignment between two scans was the most crucial part of the data processing, because this process had to be very accurate. The final feature preformed in data optimization was the fuse command, which creates automatically the mesh between the points in the point cloud and creates a watertight model as well.
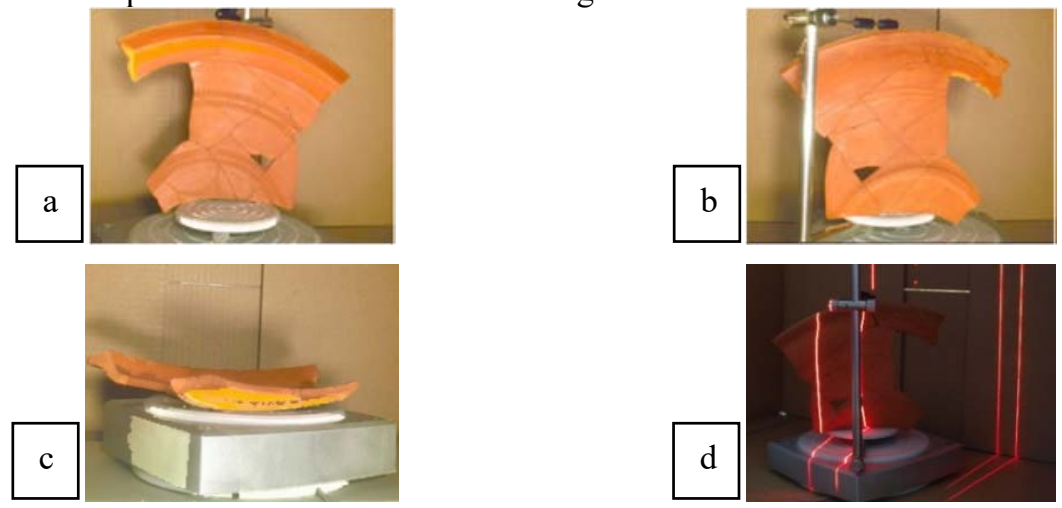

Fig. 1. Positioning of the ceramic pottery on the $360^{\circ}$ rotating base, a) pottery vertically positioned, b) pottery vertically positioned c) pottery horizontally positioned d) the actual scanning process.

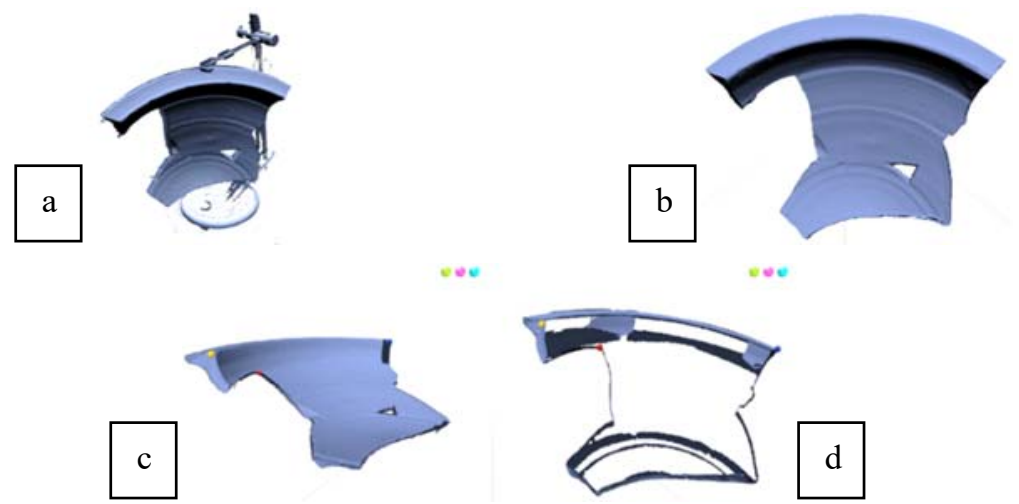

Fig. 2. The ceramic pottery a) before and b) after the elimination of extra geometries, c), d) the alignment process using the pins.

\subsection{Data processing}

The fused model that was extracted from Scan Studio contained some extra geometries that need to be deleted, and moreover the mesh of the model had to be very accurate to apply certain reverse engineering tools that Geomagic Studio provides. Some trimming and fillhole commands were used in Geomagic Studio as well, before proceeding to the simplification of the poly-faces. The simplification (named as "decimate" command) reduces the number of these faces by merging poly-vertices, while preserving geometric 
feature shapes. The decimation of the mesh was reduced to $50 \%$, which means that half of the poly-faces have been removed as illustrated in Figure 3. With the decimation of the mesh, the edges became smoother, and the "reduce noise" command was used, to produce sharper edges. This command was the last command of the "clean-up" of the mesh. The surfaces required for the mesh were generated by the "enhance mesh for surfacing" command. Although the noise was removed from the model, the mesh contained an excessive noise level. The solution on that problem was the repetition of this command (x10) (Figure 4).
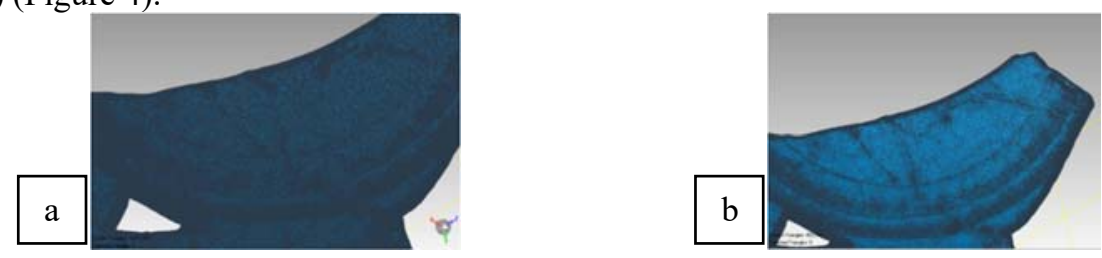

Fig. 3. The mesh model a) before and b) after the decimation of the poly-faces.
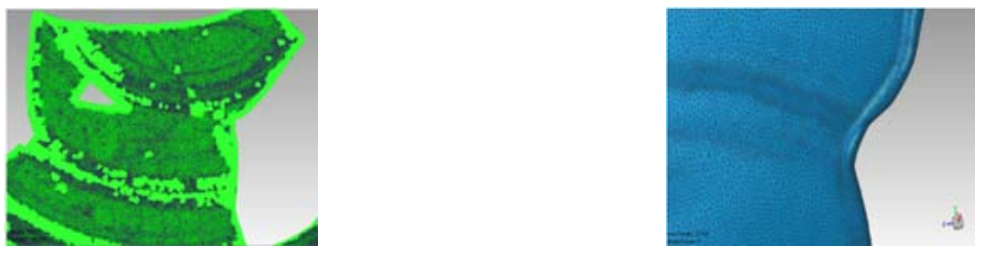

Fig. 4. Enhance mesh for surfacing that was repeated ten times.

\subsection{Reverse modelling strategy}

The reverse modelling strategy is the process of creating the best 3D model from the 3D scan data, either as just a point cloud or mesh, generated in the scanning procedure. There is a variety of different methods to follow and one of them is the "fitting surface modeling" process. Fitting surface creates a 3D freeform model based on the optimized mesh from the 3D scanned object.

The production of accurate surfaces through a scanned object requires creating an object with a good patch structure and regularly shaped patches that fill all the spaces efficiently. To achieve accurate surfaces, the detection of the contours and the creation of the actual locations of curved regions were the first two steps followed by the generation of the patches and the grids.

Finally, the surfaces were generated with the "fit surface" command, which generates a NURBS surface on the model. As shown in Figure 5, the reverse modelling strategy created a model with accurate and easy to process surfaces.
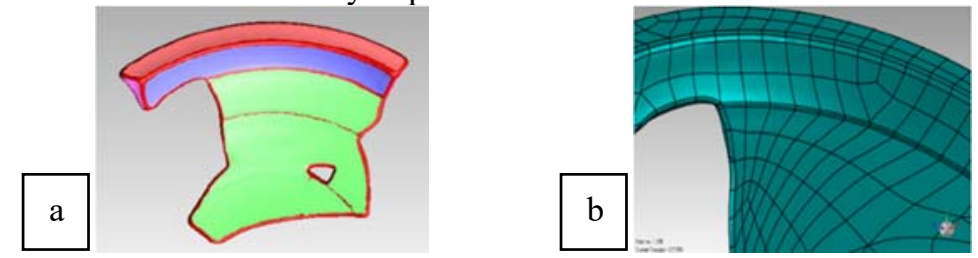

Fig. 5. a) The model that imported in Geomagic and b) the extract file after the reverse modeling strategy. 


\subsection{D restoration of the missing fragment}

The Solidworks 3D CAD software was used to create the missing fragment of the pottery. While inserting the generated surfaces from Geomagic, Solidworks identifies the surfaces and examines the presence of faulty ones. Furthermore, it automatically recognizes the features on the part. The curves that created thought the reverse modeling step are used to create a sketch, in the area that the missing fragment was supposed to be. With these curves converted into sketches and with the use of the "loft surface" command, the missing fragment was finally reconstructed. Due to the fact that were some hollow sections that created an open part, they were offset with $0 \mathrm{~mm}$ distance. This helped to create the surfaces needed and a watertight part was finally formed. Figure 6 represents the different steps followed so to create the watertight part.
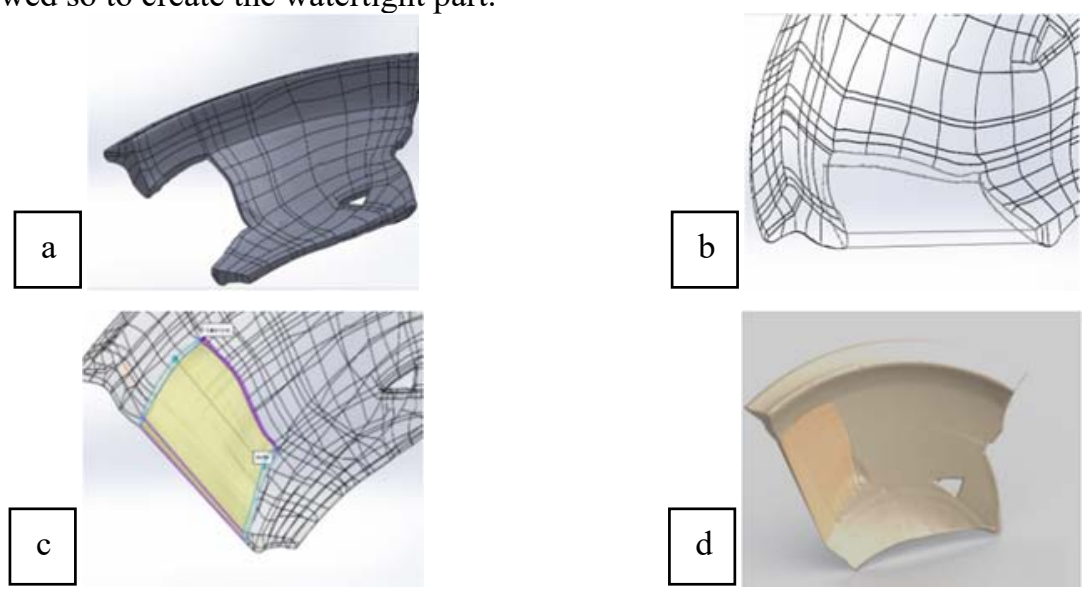

Fig. 6. The steps followed for the $3 \mathrm{D}$ reconstruction, a) recognized features, b) sketch using curves, c) loft command, d) final restored fragment.

\subsection{D printing of the missing fragment}

By following the generation of surfaces, the next step was to export the STL files for 3D printing. The generated physical model was made of white Acrylonitrile Butadiene Styrene (ABS) filament provided from ColorFabb (Netherlands). The missing fragment was 3D printed by using a BCN3D Sigma printer and Cura software as shown in Figure 7. The printing parameters used were: nozzle extrusion temperature of $210^{\circ} \mathrm{C}$, heat bed temperature of $80^{\circ} \mathrm{C}$ and printing speed of $50 \mathrm{~mm} / \mathrm{sec}$. 3D printing was carried out in a standard laboratory conditions without temperature or humidity control. The fragment was printed in vertical orientation as a monolithic shell using water soluble polyvinyl alcohol (PVA) supports in order to be easily removed.
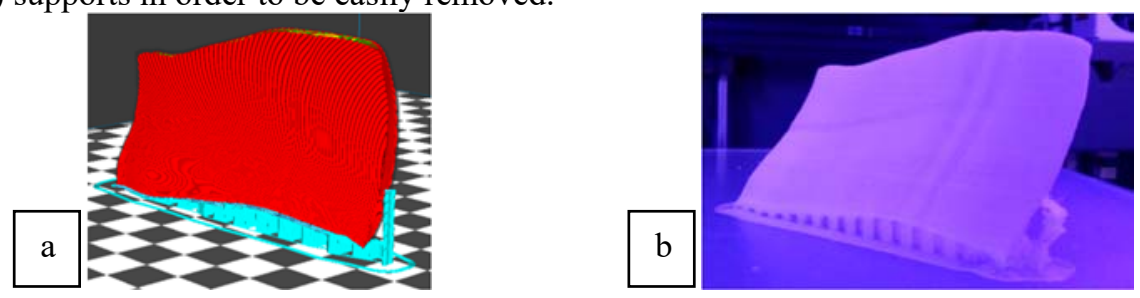

Fig. 7. a) Simulation of 3D print, b) the 3D printed fragment from ABS with the PVA support. 
Figure 8 illustrates that the digitally created fragment fits perfect the missing area of the pottery and potentially the whole ceramic pottery can be constructed with such an approach. The ABS plastic can be further surface processed with acrylic coating and colour paints so to match exactly the colour of the real archaeological find, as it has been shown by the authors in another study [4].
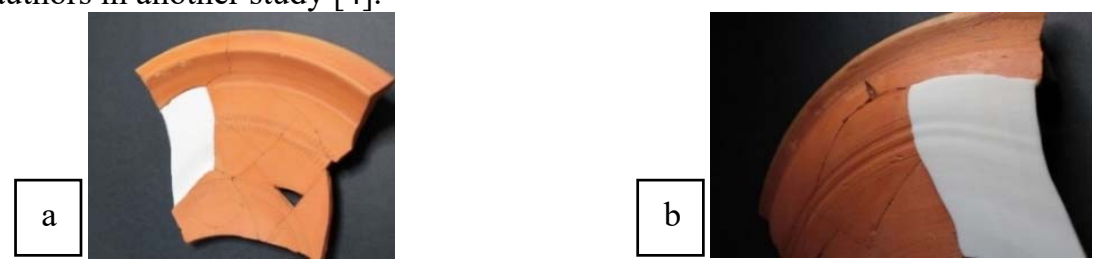

Fig. 8. a) Positioning of the 3D printed part and b) The printed part on the ceramic pottery.

\section{Conclusions}

The current paper presents the workflow of an innovative methodology for restoring missing fragments from artefacts. The visual detail of the digitally regenerated fragment was satisfactory, while the 3D printed part appears to match perfectly the missing fragment, even capturing certain surface details, such as the two lines running parallel to the outer surface of the ceramic pottery. Overall, the current work shows in a streamlined manner the process of producing digitally missing fragments of archaeological finds, which reduces the lengthy, repetitive and tedious manual restoration work that might damage further the artefact during handling. During the process followed a minimum contact with the bonded fragments of the pottery for restoration was achieved. The reconstruction effort was made by a team having experience in important functions of reverse engineering procedures, together with the use of specialised software and hardware. The work has been accomplished by using low budget equipment (desktop laser scanner), the artefact was scanned and the digitally generated CAD model was used for recreating the missing part of the highly complex geometry. The current work will further inspire the use of advanced CAD procedures in the archaeological restoration and research.

The authors would like to acknowledge Ephorate of Palaeoanthropology - Speleology of Northern Greece who gave the official permission to use the specific find for research purposes and especially Mrs. Vivi Bilali. Special acknowledgments to Xilogliptiki S.A for providing the software for the processing of the point clouds.

\section{References}

1. A. Giannelis, I. Symeonidou, D. Tzetzis, G. Kaisarlis, P. Kyratsis, MATEC Web Conference, 112 (2017)

2. G. Oancea, N.V. Ivan, R. Pescaru, AJME, 11, 4, 30 (2013)

3. Y.P. Jadhav, V.N. Chougule, A.V. Mulay, IOSRJMCE, 11 (2016)

4. A. Chatzikonstantinou, D. Tzetzis, P. Kyratsis, N. Bilalis, AMM, 657, 795 (2014)

5. N. Koumartzis, D. Tzetzis, P. Kyratsis, R.G. Kotsakis, J. New Music Res., 44, 4, 324 (2015)

6. M.J. Papagrigorakis, P.N. Synodinos, A. Antoniadis, E. Maravelakis, P. Toulas, O. Nilsson, E. Baziotopoulou-Valavani, Angle Orthod 81, 171 (2011)

7. A. Patay-Horváth, DAACH, 1, 1, 12 (2014) 